\title{
Solvent Effects on the Ultraviolet Absorption of Polystyrene
}

\author{
V. M. Story, ${ }^{*}$ D. Mclntyre, and J. H. O'Mara** \\ Institute for Materials Research, National Bureau of Standards, Washington, D.C. 20234
}

(December 21, 1966)

\begin{abstract}
NBS Standard Sample Polystyrene 705 is shown to exhibit a small, hut significant, hypochromism in the $38,300 \mathrm{~cm}^{-1}$ region in several solvents compared to the model compound, ethylbenzene. Beer's law is not obeyed for solutions of the polymer in all solvents. The concentration dependence of the extinction coefficient is related to the scattered light and its dependence on the activity coefficient of the solvent. The specific extinction coefficients for polystyrene 705 in cyclohexane and in carbon tetrachloride are 20.89 and 27.38 , respectively.
\end{abstract}

Key Words: Carbon tetrachloride, cyclohexane, ethylbenzene, extinction coefficient, polymer, polystyrene, solvent effects, ultraviolet absorption.

\section{Introduction}

The uncertainty of the concentration is further increased when several different types of experimental measurements are being made on the same polymer over a large concentration range. Some techniques like ultracentrifugal analysis use very dilute solutions; others, like osmometry use fairly concentrated solutions. The preparation of solutions for light scattering measurements involves, in addition, a filtration or centrifugation procedure which can change the concentration.

These and other conceivable difficulties were discussed before the measurement of the molecular weights of the polystyrene NBS Standard Samples 705 and 706. ${ }^{1}$ It was evident that a uniform method of determining the concentrations of existing solutions was desirable. Fortunately, the phenyl group in polystyrene is a chromophore with an appreciable specific molecular absorption in the ultraviolet. It was therefore decided to investigate the concentration dependence of the UV absorption of polystryene in various solvents before adopting this spectrophotometric analysis as a fast and accurate method of checking all solution concentrations.

A complete understanding of even small changes in spectra has become especially important in ultracentrifugation, because concentration analyses in the ultracentrifuge are now accurately made by absorption measurements at very low concentrations with photoelectric scanning. The polymer concentration can be as low as a few hundredths of one percent, and the

*Present Address: Polaroid Corporation, Cambridge, Mass. 02139.

**Present Address: Chemistry Department, Duke University, Durham, North Carolina 27706.

iSee D. McIntyre, J. Res. NBS 71 A (Phys. and Chem.) No. 1, 43 (1967), for a discussion of the polymer standard sample program. concentration may be different by a factor of three between the top and bottom of the cell. Also modern density gradient sedimentation is performed in mixed solvents with individual constituents which are often quite different in their chemical properties. Any deviation in large molecules from the spectrophotometric behavior expected of small molecules can be important in the interpretation of the centrifugation data. Concentration determinations over a hundredfold range by spectrophotometric analyses are also common in modern fractionation techniques. An implicit assumption of the validity of Beer's law for all solvents is usually made. No extensive study of the concentration dependence of the extinction coefficient of polystyrene, or other synthetic polymers, has been reported.

Shortly after the discovery of high molecular weight polystyrenes, Smakula [1] ${ }^{2}$ studied the UV absorption spectra of polystyrene dissolved in cyclohexane over a wide molecular weight and concentration range. This early work showed that the specific absorbance at 38,500 wave numbers $(2600 \AA)$ of polystyrene samples per phenyl residue is independent of the chain length of the sample and has a value only slightly less than that for the low molecular weight analog, ethylbenzene. The chain character of the molecule was shown to have little effect on either the intensity or the position of the aromatic ring absorption. In addition Smakula also found that polystyrene-cyclohexane solutions obey Beer's law in a concentration range of $0.02-0.4$ $\mathrm{g} / \mathrm{dl}$. Klevens [2] studied the UV spectra of several polymers including polystyrene in the range of 33,000 to 58,000 wave numbers ( 1720 to $3000 \AA$ ) and found that the spectra of polystyrene are different for the low

\footnotetext{
${ }^{2}$ Figures in brackets indicate the literature references at the end of this paper.
} 
molecular weight analog. He analyzed the possible causes and separated them into the following categories: (1) the incorporation of residual double bonds in the chain, (2) the enhanced light scattering, (3) the orientation and polarization of the chromophore, and (4) inter- and intra-molecular chain interactions. He concluded that polystyrene would show only the effects of scattering and possibly orientation effects.

The study of intramolecular interactions has received much attention in studies of biological macromolecules. Changes in either the optical rotation or the specific absorbance of chromophores involved in intramolecular bonding can often be interpreted in terms of the chain conformation, when other effects have been evaluated. Leach and Scheraga [3] reported a detailed study of the effects of light scattering on UV difference spectra made on large protein molecules. The correction for the light scattering effect was based on simple turbidity calculations for small, isotropic scatterers. This type of correction is easily calculated and is applicable to work on polystyrene where the molecular weights are fairly low $\left(\sim 10^{4}\right)$. Only the value of the refractive index increment is uncertain in calculating the scattering from the Rayleigh equation. The maximum error in the estimate of the turbidity can be shown to be only a fraction of a percent.

G. Loux and G. Weill [4] have also studied the effect of polymeric structure on the UV spectra of the benzene chromophore by comparing ethylbenzene and various diphenylbutane isomers to polystyrene. Their attempt to study the effect of intra- and intermolecular interactions in the polymer chain on the UV absorption by using isotactic and atactic polystyrene samples in addition to low molecular weight homologues was preceded by the theoretical and experimental study by Vala and Rice [5] of the UV spectra of isotactic and atactic polystyrene. Vala and Rice interpreted their results by postulating an average ordered orientation of neighboring phenyl groups in the isotactic polystyrene molecule in solution. The present study, in addition to establishing values of the extinction coefficients, also was aimed at understanding the solventsolute interactions as they affect the UV spectra. These effects are very difficult to predict, since the solvent can act strongly enough to form a charge transfer complex or weakly enough to just keep the polymer dissolved and thus encourage segment-segment contacts. For these reasons three solvents were used: (1) a very good solvent (carbon tetrachloride), (2) a good solvent (tetrahydrofurane), (3) and a moderate to poor solvent (cyclohexane at various temperatures).

\section{Experimental Procedure}

\subsection{Materials}

Polymer.-NBS Standard Sample Polystyrene-705, having a narrow molecular weight distribution, was used. This polymer has a number-average molecular weight of 170,900 (osmotic pressure) and a weightaverage molecular weight of 179,300 (light scattering). The sample was prepared by the polymerization of styrene in benzene using $n$-butyllithium as an initiator.
The polymer was dissolved in benzene and freezedried before use. High-vacuum experiments above the glass temperature on polymer samples treated in this way show that the remaining solvent content is less than 0.5 percent. Unless otherwise stated, the solutions were made from freeze-dried polymer.

Ethylbenzene. - A research-grade sample of ethylbenzene, whose mole-percent purity was stated to be 99.57 as determined by melting point measurements, was used.

Solvents.-Reagent-grade cyclohexane was distilled through a column packed with glass helices. Spectro grade carbon tetrachloride and tetrahydrofurane were used as received.

\subsection{Preparation of Solutions}

All solutions were independently prepared on a weight basis using from 21 to more than $100 \mathrm{mg}$ of polymer or ethylbenzene for each independent solution. All experimental concentrations are expressed in $\mathrm{g} / \mathrm{dl}$. The concentrations were computed for the given temperatures by use of the appropriate solvent densities, since the solutions were very dilute.

\subsection{Spectrophotometer}

A commercial recording spectrophotometer was used in this work. The wavelength resolving power was $\sim 1 \AA$, and the wavelength reproducibility better than $\sim 0.5 \AA$ in the spectral region measured. The scan speed used was $0.5 \AA$ per second.

Spectra were obtained in the 43,500 to 34,500 wavenumber region except in the case of $\mathrm{CCl}_{4}$ solutions for which spectra were obtained in the 40,000 to 34,500 wave-number region. Higher frequencies could not be scanned with $\mathrm{CCl}_{4}$ as solvent because of the intense solvent band above 40,000 wave numbers.

Two independent spectral runs were made on each solution and the absorption values averaged. The reproducibility was 0.2 percent or better.

\subsection{Cells}

All cells, either fixed-bath length of $10 \mathrm{~mm}$ or $5 \mathrm{~mm}$, or a variable thickness cell, were fitted with ground glass stoppers. The solution and solvent cells were placed in a thermostated cell compartment, and the temperature was regulated by circulating water through the cell compartments from a thermostated circulating bath placed just outside the spectrophotometer. The temperature of the cell was measured after 15 min or more had been allowed for equilibration. At settings in the circulating bath of $20,35,45$, and $55^{\circ} \mathrm{C}$, the thermocouple readings in the sample cell gave temperature readings of $19.88,34.57,44.56$, and 54.09 $\pm 0.01{ }^{\circ} \mathrm{C}$, respectively.

\section{Results}

Figure 1 compares the spectra for the 38,500 wave number $(2600 \AA)$ region of ethylbenzene and polystyrene 705 in cyclohexane. Figure 2 presents the corresponding spectra in carbon tetrachloride. The 


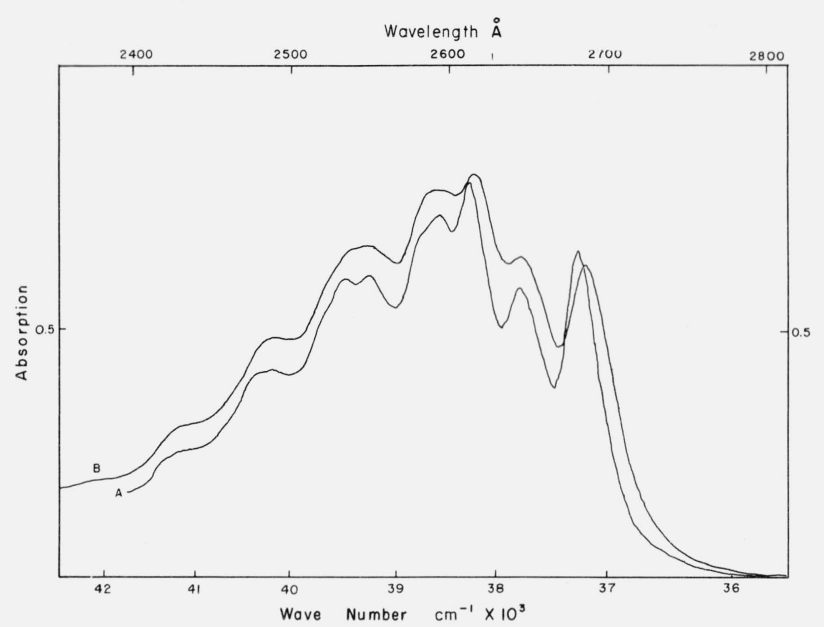

FigURE 1. * Curve A; ethylbenzene in cyclohexane at $35^{\circ} \mathrm{C}$, concentration $0.03746 \mathrm{~g} / \mathrm{dl}$. $B$; polystyrene 705 in cyclohexane at $35^{\circ} \mathrm{C}$, concentration $0.04015 \mathrm{~g} / \mathrm{dl}$.

*Absorption spectra at various wavelengths.

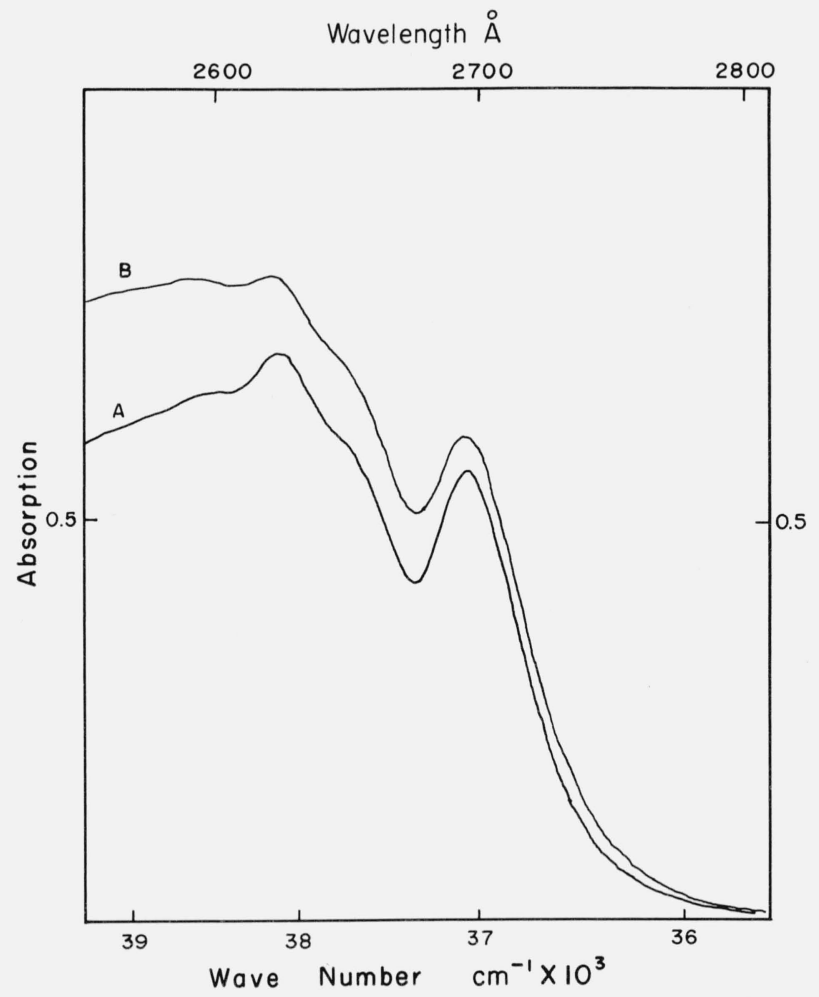

Figure 2. *Curve A; ethylbenzene in carbon tetrachloride at $35^{\circ} \mathrm{C}$, concentration $0.02576 \mathrm{~g} / \mathrm{dl}$. $B$; polystyrene 705 in carbon tetrachloride at $35^{\circ} \mathrm{C}$, concentration $0.03188 \mathrm{~g} / \mathrm{dl}$.

*Absorption spectra at various wavelengths. concentrations of the solutions are about $0.03 \mathrm{~g} / \mathrm{dl}$, and the temperature is $35^{\circ} \mathrm{C}$.

Tables 1 and 2 list the data for linear and quadratic fits of absorption (A) versus the concentration (c) for ethylbenzene and polystyrene-705, respectively, in cyclohexane and carbon tetrachloride at $35^{\circ} \mathrm{C}$. Table 3 reports some earlier data taken over a smaller concentration range. The experiments reported in table 3 , which also includes a third solvent, were undertaken to determine if there was a significant effect of temperature or solvent on the extinction coefficient. The absorbance is fitted to a linear function of concentration, since only a few determinations over a small concentration range are available in some instances.

Table 4 compares the wavelength shift of the $\nu_{\max }$ of polystyrene and ethylbenzene. Also the extinction

TABLE 1. Spectral data for ethylbenzene

\begin{tabular}{|c|c|c|}
\hline & $\mathrm{C}_{6} \mathrm{H}_{12}$ & $\mathrm{CCl}_{4}$ \\
\hline$T^{\circ} \mathrm{C}$ & 35 & 35 \\
\hline Conc. $\}$ & 0.025 & 0.0257 \\
\hline Range $\} \mathrm{g} / \mathrm{al}$ & .089 & .0467 \\
\hline & 13 & 6 \\
\hline$\nu_{\max } \mathrm{cm}^{-1}$ & 38,310 & 38,170 \\
\hline Linear fit ${ }^{* *}$ & & \\
\hline $\begin{array}{l}a_{1} \pm S_{a_{1}} \\
S \times 10^{3}\end{array}$ & $\begin{array}{l}21.78 \pm 0.04 \\
7.774\end{array}$ & $\begin{array}{l}28.00 \pm 0.002 \\
\quad 1.975\end{array}$ \\
\hline Quadratic fit** & & \\
\hline $\begin{array}{l}a_{1} \pm S_{a_{1}} \\
a_{2} \pm S_{a_{2}} \\
S \times 10^{3}\end{array}$ & $\begin{array}{l}21.77 \pm 0.01 \\
1.542 \pm 1.978 \\
8.086\end{array}$ & $\begin{array}{l}27.80 \pm 0.01 \\
0.051 \pm 0.026 \\
1.578\end{array}$ \\
\hline
\end{tabular}

$* N$ is the number of determinations.

${ }^{* *} a_{1}$ and $a_{2}$ are coefficients determined by least squares fits of the equations $A=a_{1} c$ and $A=a_{1} c+a_{2} c^{2} . \quad S_{a_{i}}$ are the standard deviations of the coefficients of the linear or quadratic fits. $S$ is the standard deviation of the fit.

TABLE 2. Spectral data for polystyrene-705 (extended concentration range)

\begin{tabular}{|c|c|c|}
\hline & $\mathrm{C}_{6} \mathrm{H}_{12}$ & $\mathrm{CCl}_{4}$ \\
\hline$T{ }^{\circ} \mathrm{C}$ & 35 & 35 \\
\hline Conc. \} & 0.022 & 0.025 \\
\hline Range $\} \mathrm{g} / \mathrm{dl}$ & .194 & .154 \\
\hline & 14 & 13 \\
\hline$\nu_{\max } \mathrm{cm}^{-1}$ & 38,270 & 38,200 \\
\hline Linear fit ${ }^{* *}$ & & \\
\hline $\begin{array}{r}a_{1} \pm S_{a_{1}} \\
S \times 10^{3}\end{array}$ & $\begin{array}{l}20.89 \pm 0.08 \\
10.20\end{array}$ & $\begin{array}{l}27.05 \pm 0.07 \\
11.06\end{array}$ \\
\hline Quadratic fit** & & \\
\hline $\begin{array}{r}a_{1} \pm S_{a_{1}} \\
a_{2} \pm S_{a_{2}} \\
S \times 10^{3}\end{array}$ & $\begin{array}{l}20.97 \pm 0.03 \\
0.549 \pm 0.574 \\
10.16\end{array}$ & $\begin{array}{l}27.38 \pm 0.07 \\
-3.347 \pm 0.646 \\
\quad 6.97\end{array}$ \\
\hline
\end{tabular}

$* N$ is the number of determinations.

$* * a_{1}$ and $a_{2}$ are coefficients determined by the equation $A=a_{1} c$ $+a_{2} c^{2} . \quad S_{a_{i}}$ is the standard deviation of the coefficient of the linear or quadratic fit. 
TABLE 3. Spectral results for polystyrene-705 (short concentration range)

\begin{tabular}{l|c|c|c|r|r|r|r|r}
\hline \hline Solvent & Temp. & \multicolumn{2}{|c|}{ Concentration range } & $N$ & $\nu_{\max }$ & $a_{1}$ & $S_{a_{1}}$ & $S \times 10^{3}$ \\
\hline & ${ }^{\circ} \mathrm{C}$ & & & & $c m^{-1}$ & & & \\
$\mathrm{C}_{6} \mathrm{H}_{12}$ & 20 & 0.021 & 0.081 & 7 & 38,270 & 20.68 & 0.06 & 6.7 \\
& 35 & .022 & .097 & 10 & 38,270 & 20.45 & .11 & 10.5 \\
& 45 & .026 & .093 & 6 & 38,270 & 20.25 & .12 & 10.2 \\
& 55 & .020 & .091 & 8 & 38,270 & 20.47 & .04 & 7.3 \\
$\mathrm{C}_{4} \mathrm{H}_{8} \mathrm{O}$ & 35 & .03 & .09 & 11 & 38,260 & 21.01 & .04 & 8.0 \\
$\mathrm{CCl}_{4}$ & 35 & .025 & .071 & 8 & 38,200 & 26.18 & .05 & 7.0 \\
& 55 & .025 & .072 & 5 & 38,200 & 25.57 & .04 & 4.1 \\
\hline
\end{tabular}

TABLE 4. Comparison of ethylbenzene and polystyrene-705 at $35^{\circ} \mathrm{C}$

\begin{tabular}{c|c|c|c}
\hline \hline & $\begin{array}{c}(1) \\
\text { Ethylbenzene }\end{array}$ & $\begin{array}{c}(2) \\
\text { Polystyrene-705 }\end{array}$ & $\begin{array}{c}\text { Difference } \\
(1)-(2)\end{array}$ \\
\cline { 2 - 4 } $\mathrm{C}_{6} \mathrm{H}_{12}$ \\
\hline$\nu_{\max } \mathrm{cm}^{-1}$ & 38,310 & 38,270 & \\
$a_{1}$ & 21.78 & 20.89 & +40 \\
$a_{2}$ & 0 & 0 & +0.89 \\
$\frac{\mathrm{CCl}_{4}}{\nu_{\max } \mathrm{cm}^{-1}}$ & 38,170 & 38,200 & \\
$a_{1}$ & 28.00 & 27.38 & -30 \\
$a_{2}$ & 0 & -3.35 & +0.62 \\
$\mathrm{C}_{6} \mathrm{H}_{12}-\mathrm{CCl}_{4}$ & & & \\
\hline$\Delta \nu_{\max }$ & +140 & +70 & \\
$\Delta a_{1}$ & -6.22 & -6.49 & \\
\hline
\end{tabular}

coefficients, $a$, determined from the best fit of the data are intercompared.

\section{Errors}

The uncertainties in measuring peak intensities are approximately \pm 0.004 absorption units, while the wave numbers can be measured to within $\pm 15 \mathrm{~cm}^{-1}$. The solution concentration errors are (1) errors in weighing the solute, (2) errors in weighing the solvent, and (3) unknown changes in the concentration due to the evaporation of the solvent. The last error was minimized by weighing the solutions immediately before making the spectrograms. Evaporation of solvent from the stoppered cells is not significnat, since the duplicate spectra taken agreed within instrumental error.

The maximum weighing error for the polymer and ethylbenzene is about 1 percent, while that for the solvent was less than 0.1 percent (bouyancy corrections were made because large volumetric flasks were used). The errors in the determination of the extinction coefficient at a given wavelength were minimized by measuring only those solutions whose absorbance was between 0.3 and 1.7. Under these conditions, the maximum error in an extinction coefficient determined from one concentration is about 1 percent. The effect of small temperature changes on the absorption maxima in the electronic ultraviolet spectra would be expected to be negligible, although the concentration would be directly affected. The solution temperatures were controlled to within $\pm 0.01{ }^{\circ} \mathrm{C}$. Densities, and hence concentrations, change less than 0.1 percent with the above temperature variations in the solvents.
Measurable turbidities existed in the polystyrene solutions. Small increases of the extinction coefficient, which increased with increasing concentration and increase in wave number, appeared in regions where ethylbenzene showed no absorption. Filtration of the polystyrene solution through an 0.8-micron millipore filter demonstrated that the observed turbidity was not due to foreign particles. The effect of the turbidity on the absorbance of the solutions is treated later in the analysis of the absorption data for polystyrene solutions.

Another potential source of error in this type of measurement is the change of slit width required as the concentration of the solute is increased. Empirical calibration curves were determined to correct the absorption measurements for the solutions of ethylbenzene and polystyrene in cyclohexane and in carbon tetrachloride. For the slit widths observed, the ratio of the slit width, $s$, to the half peak width was estimated to be less than 0.15. For slit widths below the value corresponding to this ratio, slit-width corrections are, to a first approximation, linear [6]. These corrections were small, averaging less than \pm 0.3 percent. They were less than the instrumental errors in the experiments reported in table 3 , and were not applied to these results.

The dependence of absorption, A, on concentration, c, for polystyrene solutions can be either linear or quadratic. The quadratic dependence is required when the solvent is not an ideal solvent because the turbidity then has a quadratic dependence on concentration. Allowing a maximum error of 1 percent for instrumental reproducibility, and a maximum error of 1 percent in the concentration, the overall errors might be as high as 2 percent. Therefore 95 percent confidence limits were used as the statistical criterion for deciding whether the linear or quadratic best fit the measurements of the UV absorption of polystyrene as a function of concentration in cyclohexane and carbon tetrachloride (table 1) [7].

\section{Discussion}

The spectra of ethylbenzene and polystyrene in cyclohexane and carbon tetrachloride are shown in figures 1 and 2, respectively. The polystyrene spectra have much less detail than the ethylbenzene spectra in the same solvent. The cyclohexane fortunately does not have an absorption edge at as low a frequency as carbon tetrachloride. However, even in carbon tetrachloride the spectral region for the forbidden electronic transition of the phenyl group $\left(\pi^{*} \leftarrow \pi\right)$ centering at $2600 \AA$ is still accessible experimentally. This transition is not as strongly affected by solvents as the more intense, higher frequency transition [2, 8], and therefore is of considerable value in analytical chemistry.

\subsection{Frequency Changes}

Although the frequency shifts are expected to be small in comparing ethylbenzene to an atactic poly- 
styrene, the shifts are nevertheless significant. Loux and Weill [4] have shown that 1,4-diphenylbutane has a spectrum almost identical to that of ethylbenzene; whereas 1,3-diphenylbutane has a spectrum that modifies the ethylbenzene spectrum to be almost identical to that of atactic polystyrene. Loux and Weill do not mention any displacement of the various maxima. However, the data in table 4 of this paper clearly show that the frequency shift of the maximum for polystyrene compared to ethylbenzene is small, but beyond experimental error, and can be of a different sign. Thus in cyclohexane, and presumably in most nonpolar solvents, the shift for a polymer compared to its repeating unit is towards the red. But in strongly interacting solvents, like carbon tetrachloride, there is a very small shift of the maximum peak to the blue. The frequency shifts reported in table 4 for both ethylbenzene and polystyrene in proceeding from cyclohexane to carbon tetrachloride are towards the red, $\sim 100 \mathrm{~cm}^{-1}$. These results are in agreement with the results of Bayliss and Hulme [8] who found a similar red shift for toluene of $170 \mathrm{~cm}^{-1}$ in cyclohexane and carbon tetrachloride. Table 3 indicates that small changes in the solvent environment caused by a change of temperature or enhanced, but nondirected, solvation do not affect the maximum frequency.

\subsection{Intensity Changes}

The best measure of the change of the strength of the electronic transition is an estimate of the oscillator strength. For analytical purposes this quantity is not needed, and the data in tables 1 to 4 only represent the extinction coefficient of the absorption maximum at the reported frequency, $\sim 38,300 \mathrm{~cm}^{-1}$.

Recent interest in macromolecular structure has centered on intramolecular interactions and their influence on spectral absorption. Vala and Rice [5] found experimentally that there is a 10 percent hypochromic change in the $2600 \AA$ band, when isotactic polystyrene is compared to atactic polystyrene dissolved in the same solvent, chloroform. This value is in good agreement with the theoretical value of 12.8 percent which they calculated on the basis of the crystalline structure of isotactic polystyrene. The calculated hypochromism is based upon nonspecific solvation which decreases the intensity of absorption for the ordered isotactic polystyrene compared to the atactic polystyrene. The measured hypochromism appears to be influenced by the type of solvent because the effect in dioxane and dichloromethane is 4.5 and 3.3 percent, respectively.

The values of $a$ for ethylbenzene and polystyrene in table 4 show a decrease in the extinction coefficient of 5 percent in cyclohexane and 2 percent in carbontetrachloride. The change of $a$ in any solvent which does not have a specific interaction, like tetrahydrofurane and cyclohexane, is almost negligible, as shown in table 3. Loux and Weill had reported a 1 to 5 percent hyperchromic effect in comparing polystyrene and ethylbenzene in tetrahydrofurane, cyclohexane, and decalin, based on oscillator strengths. The results reported here, based only on the $a_{\max }$, are of the same magnitude but are hypochromic. In contrast the polystyrene spectra in tetrahydrofurane reported by Loux and Weill show a polystyrene peak larger than the ethylbenzene peak at $2600 \mathrm{~A}$. The hypochromism of polystyrene compared to ethylbenzene is reported by Loux and Weill to be 3 to 4 percent in chloroform, and the results in table 4 for carbon tetrachloride solutions is 2 percent, or about the same effect. Changes in the virial coefficient, effected by increasing the cyclohexane temperature or changing to tetrahydrofurane, show very little change in the $a_{\max }$, less than 3 percent.

The recent work of Goates, Sullivan, and Ott [10] has demonstrated the existence of a one-to-one complex between benzene and carbon tetrachloride by measurement of the freezing point curves. They obtained similar results for the $p$-xylene-carbon tetrachloride system, and calculated the heats of dissociation to be 3.5 and $6.3 \mathrm{kcal} / \mathrm{mole}$, respectively. No complex was evident when the second component was chloroform. Nitrobenzene and carbon tetrachloride mixtures also failed to form a detectable complex. Goates, Sullivan, and Ott concluded that electron withdrawal due to substituents in the benzene ring prevent the formation of the complex.

The same conclusions for the existence of chemical complexes in the benzene-carbon tetrachloride solutions are supported by other studies of charge-transfer complexes between the empty $d$ orbitals of halogen compounds and $\pi$ electron systems [9]. Bayliss and Hulme found a slow increase in the extinction coefficient of toluene for solvents progressing from highly polar solvents like ethanol to solvents like cyclohexane and chloroform. In chloroform relative to cyclohexane the increased absorption is only 3 percent, while the relative increase in carbon-tetrachloride is 40 percent. Both for polystyrene and ethylbenzene the same relative increase of $\epsilon_{\max }$ reported in table 4 is $\sim 28$ percent.

In conclusion, if only weakly interacting solvent systems are considered, the changes in the spectra are very small. The hypochromism of isotactic polystyrene compared to atactic polystyrene is no greater than 3 to 4 percent [5]. Also the hypochromism of atactic polystyrene compared to ethylbenzene is not greater than 5 percent. This latter change is comparable in magnitude to the change of spectra reported by Klevens for 1,3 diphenylpropane, and by Loux and Weill for 1,3 diphenylbutane. A much larger spectral change in the polystyrene spectra can be induced by solvents like carbon tetrachloride which are capable of forming charge transfer complexes. The role of these solvents in polymer conformation is largely unknown. The hypochromic shift in chloroform reported by Vala and Rice might even be larger with carbon tetrachloride.

The data of table 4 and the observations of Goates, Sullivan, and Ott indicate that, to a very high degree, all of the phenyl rings are available for solvation by carbon tetrachloride. This seems startling since some of the rings must be somewhat inaccessible due to the polymer conformation. However, ethylbenzene and 
polystyrene solutions in carbon tetrachloride have the same percentage change in $\epsilon_{\max }$ compared to a reference state of cyclohexane solutions. The polymer environment apparently is sufficiently constrained to cause a decrease intensity, as evidenced by a comparison of the ethylbenzene and polystyrene values of $a_{\max }$ in any solvent.

\subsection{Concentration Dependence and Light Scattering}

The absorbance of ethylbenzene is best fitted as a linear function of concentration over the extended concentration ranges. Tables 1 and 2 give the parameters for the least squares fitting of the absorbance for both cyclohexane and carbon tetrachloride solutions, $N$ is the number of concentrations and $S$ is the standard deviation. The statistical data were calculated by the Omnitab computer program [11]. Polystyrene in cyclohexane also gives a best fit when the absorbance is considered to be a linear function of concentration. The absorbances of polystyrene in carbon tetrachloride at $35^{\circ} \mathrm{C}$ are best represented by a quadratic equation, with the quadratic coefficient being negative. Only over the extended range of concentration is it possible to detect the negative term. As the data in table 3 indicate, the negative curvature does not show up in the measurements at low concentrations. It is conceivable that the conformational changes in the polymer do change as the concentration is increased. Certainly the overall dimensions are known to change, but the data in tables 1,2 , and 3 indicate that the effect is so small that it is not easily seen.

If there is a quadratic dependence of absorbance on concentration, then Beer's law is not obeyed. Almost all chemical systems do obey Beer's law, unless there is a chemical change occurring as concentration is increased. Before any decision on the inapplicability of Beer's law to macromolecules is made in a macromolecular system, the scattering turbidity must be carefully analyzed and subtracted from the general absorbance. The attenuation of the irradiating light beam can be due to either of two simple processes, simple molecular absorption, or scattering of the electromagnetic radiation. It is difficult to separate the two effects by transmission measurements at a fixed wavelength, but independent measurements of of the transverse molecular scattering are possible.

Consider a light beam of incident irradiance, $I_{0}$, that is attenuated in its passage through the sample of length, $l$, and concentration, $c\left(\mathrm{~g} / \mathrm{cm}^{3}\right)$, to irradiance, $I$. The transmittance, $\left(I / I_{0}\right)$, or the absorbance, $(A)$, may be written in terms of the specific extinction coefficient, $\epsilon$, and turbidity, $\tau$, as follows:

$$
A=-\ln \frac{I}{I_{0}}=\epsilon c l+\tau l .
$$

The turbidity may be expressed as a function of the chemical potential of the solvent $(\mu)$, the refractive index $(n)$, of the solution, the wavelength $(\lambda)$, and Avogadro's number $(N)$ as

$$
\tau=\frac{32 \pi^{3}}{3 \lambda^{4}} \frac{n^{2}(d n / d c)^{2}}{-\left(\frac{\partial \mu}{\partial c}\right)}
$$

Since the chemical potential of solvent can be expressed as a linear function of the virial coefficient $\left(A_{2}\right)$ in a dilute solution, the above expression becomes

$$
\tau=\frac{32 \pi^{3}}{3 \lambda^{4}} \frac{n^{2}(d n / d c)^{2} c M}{N\left(1+2 A_{2} c\right)}=\frac{H c M}{\left(1+2 A_{2} c\right)} \cong H c M\left(1-2 A_{2} c\right) .
$$

Therefore the absorbance per centimeter in dilute solutions may be written as

$$
\begin{aligned}
A & =\epsilon c+H c M\left(1-2 A_{2} c\right) \\
& =(\epsilon+H M) c-2 A_{2} H M c^{2}=c\left[\epsilon+\tau_{0}\left(1-2 A_{2} c\right)\right] \\
& \equiv a_{1} c+a_{2} c^{2} .
\end{aligned}
$$

For polystyrene-705 the contribution of the scattering at $2600 \AA$ to the absorbance may be estimated, since values of $M, H$, and $A_{2}$ are known. For cyclohexane at $35{ }^{\circ} \mathrm{C}, A_{2}$ has been measured and is zero. In this special case there should be no deviation from Beer's law. For cyclohexane below $35^{\circ} \mathrm{C}, A_{2}<0$; for cyclohexane $>35{ }^{\circ} \mathrm{C}$, tetrahydrofurane and carbon tetrachloride, $A_{2}>0$. An estimate of $A_{2}$ based on osmotic pressure measurements of polystyrene-carbon tetrachloride solutions of a similar molecular weight polystyrene is $64 \times 10^{-5}$ in cgs units. An estimate of $(d n / d c)$ for cyclohexane is $\sim 0.34 \mathrm{~cm}^{3} / \mathrm{g}$ for $2600 \AA$ [12]. An estimate based on a $\lambda^{-2}$ dependence of $d n / d c$ would give a smaller value for carbon tetrachloride. An estimate of the excess scattering in cyclohexane solutions for a polymer of molecular weight of 180,000 would correspond to a turbidity of $0.57 \mathrm{c}$, or 2 percent of the total absorbance of a 0.1 percent solution at $2600 \AA$. An estimate of the excess molecular scattering of carbon tetrachloride solutions based on a comparable change of $d n / d c$ for carbon tetrachloride as exists in cyclohexane is approximately 1.5 times less than the cyclohexane solution scattering. At the low concentrations used in these experiments (0.1 percent or less), the contribution of the virial coefficient to the total absorbance would be less than 0.4 percent. In the form suggested by eq (3), the correction for the absorbance in carbon tetrachloride would be

$$
A=A_{0} c-1.63 \mathrm{c}^{2}
$$

The value of the quadratic term reported in table 4 is -3.35 . This is greater than the estimated value and probably does indicate a small deviation of Beer's law. Whether this deviation can be attributed to a squeezing out of segment-solvent interactions by a crowding of the phenyl groups is of course quite speculative. The dependence on concentration in tetrahydrofurane ought to be about the same as in carbon tetrachloride because the virial coefficient is smaller, while the $(d n / d c)$ is larger. 


\subsection{Extinction Coefficients of Polystyrene 706}

A single quartz prism spectrophotometer was used to measure the extinction coefficient of polystyrene 706 at $2618 \AA$. The solutions were made from the pellet form of the polymer. The extinction coefficient was determined to be $24.03 \pm(0.08)$ on the basis of three determinations at different concentrations. This extinction coefficient is considerably higher than that of pure polystyrene. The high extinction coefficient is probably due to a monomer impurity. (See footnote 1.) By using the equations of Newell [13], who assumed the presence of only two components (styrene monomer and polymer) in the mixture, the monomer content is estimated to be 0.33 percent. The estimates of volatiles given in the first paper of this series are 0.82 percent. (See footnote 1.) No further attempt has been made to determine the source of this difference. Some of the volatiles are low molecular weight material. A future publication of this series will discuss the characteristics of the low molecular weight fractions.

\section{References}

[1] A. Smakula, Angewandte Chemie 47, 777 (1931).

[2] H. B. Klevens, J. Polymer Sci. 10, 97 (1953).

[3] S. J. Leach and H. A. Scheraga, J. Am. Chem. Soc. 82, 4790 (1960).

[4] G. Loux and G. Weill, J. Chem. Phys. 61, 484 (1964).

[5] M. T. Vala, Jr. and S. A. Rice, J. Chem. Phys. 39, 2348 (1963).

[6] R. N. Jones and C. Sandorfy, in Techniques of Organic Chemistry, Vol. IX, edited by W. West, Interscience, New York. 1965. pp. 279-284 and references listed there.

[7] Experimental Statistics, by M. Natrella, NBS Handbook 91, U.S. Government Printing Office, 1963; par. 5-1 et. seq.

[8] N. S. Bayliss and L. Hulme, Australian J. Chemistry, 6, 257 (1953).

[9] Electronen-donator-acceptor-komplexe, G. Briegleb (Springer Verlag, 1961); p. 31, table 10.

[10] J. R. Goates, R. J. Sullivan, and J. B. Ott, J. Phys. Chem. 63, 589 (1959).

[11] J. Hilsenrath, G. G. Ziegler, C. G. Messina, P. J. Walsh, and R. J. Herbold, Omnitab, Chap. 5, NBS Handbook 101, 1966.

[12] P. Peyser, private communication.

[13] J. E. Newell, Anal. Chem. 23, 444 (1951).

(Paper 71A2-449) 